\title{
G

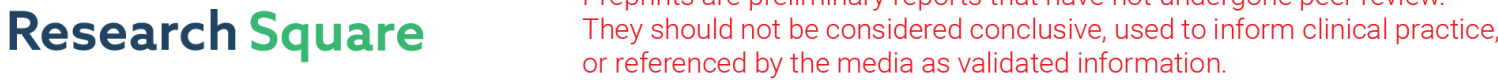 \\ The Association between Urine Elements and Fasting Glucose Levels in a Community-Based Elderly People in Beijing
}

\section{Liu Liu}

Chinese Center for Disease Control and Prevention National Institute of Environmental Health

\section{Ang Li}

Peking Union Medical College School of Basic Medicine: Chinese Academy of Medical Sciences and Peking Union Medical College Institute of Basic Medical Sciences

\section{Qun Xu}

Peking Union Medical College School of Basic Medicine: Chinese Academy of Medical Sciences and Peking Union Medical College Institute of Basic Medical Sciences

\section{Qin Wang}

Chinese Center for Disease Control and Prevention National Institute of Environmental Health

\section{Feng Han}

Chinese Center for Disease Control and Prevention National Institute of Environmental Health

\section{Chunyu Xu}

Chinese Center for Disease Control and Prevention National Institute of Environmental Health

\section{Zhe Liu}

Chinese Center for Disease Control and Prevention National Institute of Environmental Health

\section{Dongqun Xu ( $\nabla$ xudq@chinacdc.cn)}

Chinese Center for Disease Control and Prevention National Institute of Environmental Health

\section{Donggang Xu ( $\sim$ xudg@bmi.ac.cn)}

Beijing Institute of Basic Medical Sciences

\section{Research Article}

Keywords: Co-existing element, Fasting blood glucose, Diabetes mellitus, Urine, Bayesian kernel machine regression

Posted Date: February 24th, 2021

DOI: https://doi.org/10.21203/rs.3.rs-235017/v1

License: (c) (i) This work is licensed under a Creative Commons Attribution 4.0 International License. Read Full License 
Version of Record: A version of this preprint was published at Environmental Science and Pollution Research on January 8th, 2022. See the published version at https://doi.org/10.1007/s11356-021-179488. 


\section{Abstract}

Epidemiological studies have demonstrated that various kinds of urinary element concentrations were different between healthy, prediabetes, and diabetes patients. Meanwhile, many studies have explored the relationship between element concentration and fasting blood glucose (FBG), but the association between joint exposure to co-existing elements and FBG level has not been well understood. The study explored the associations of joint exposure to co-existing urinary elements with FBG level in a crosssectional design. 275 retired elderly people were recruited from Beijing, China. The questionnaire survey was conducted, and biological samples were collected. The generalized linear model (GLM) and twophase Bayesian kernel machine regression (BKMR) model were used to perform in-depth association analysis between urinary elements and FBG. The GLM analysis showed that $\mathrm{Zn}, \mathrm{Sr}$, and $\mathrm{Cd}$ were significantly correlated with the FBG level, under control potential confounding factors. The BKMR analysis demonstrated 8 elements ( $\mathrm{Zn}, \mathrm{Se}, \mathrm{Fe}, \mathrm{Cr}, \mathrm{Ni}, \mathrm{Cd}, \mathrm{Mn}$, and $\mathrm{Al}$ ) had a higher influence on FBG (Posterior inclusion probabilities $>0.1$ ). Further intensive analyses result of the BKMR model indicated that the overall estimated exposure of 8 elements was positively correlated with the FBG level and was statistically significant when all element concentrations were at their 65 th percentile. Meanwhile, the BKMR analysis showed that $\mathrm{Cd}$ and $\mathrm{Zn}$ had a statistically significant association with FBG levels when other co-existing elements were controlled at different levels (25th, 50th or 75th percentile), respectively. The results of the GLM and BKMR model were inconsistent. The BKMR model could flexibly calculate the joint exposure to co-existing elements, evaluate the possible interaction effects and nonlinear correlations. The meaningful conclusions were found that it was difficult to get by traditional methods. This study will provide methodological reference and experimental evidence for the association between joint exposure to co-existing elements and FBG in elderly people.

\section{Introduction}

Type 2 diabetes mellitus (T2DM) is an endocrine and metabolic disease characterized by a disturbance of blood glucose, protein, and lipid metabolism disturbance(Henning 2018). Globally, T2DM is a major threat to human health worldwide and may result in an increased incidence and high prevalence. According to the World Health Organization (WHO), there were 422 million diabetes patients, with $80 \%$ of them belonging to low and middle-income countries (Jaacks et al. 2016; WHO 2021). The International Diabetes Federation (IDF) estimated that the number of patients with diabetes was about 116 million in China. Further investigation revealed that the number of patients with diabetes in China had increased by $62.7 \%$ over the past decade, and it is one of the countries with the highest estimated number of patients with diabetes, accounting for nearly $30 \%$ of the total world (Liu et al. 2019; Williams 2021). With the increases in the size of the aging population, changes in lifestyle, economic and environmental factors, the prevalence of T2DM is increasing consistently.

Epidemiological studies have recently shown that transportation, distribution, excretion, and accumulation of various kinds of elements, including $\mathrm{Zn}, \mathrm{Cu}, \mathrm{Se}$, and As, differ in prediabetes, and diabetes patients relative to healthy individuals (Menke et al. 2016; Sanjeevi et al. 2018; Wang et al. 
2020a). This is because many elements are involved in various physiological functions and energy metabolisms in mammals and play a corresponding role as well (Fiore et al. 2020). This suggests that various physiological states may be associated with altered element levels. However, findings from these studies remain controversial (Hansen et al. 2017; Kohler et al. 2018).

In environmental health research, the prevalent tool to evaluate the association between elements exposure and health effects has been the generalized linear model (GLM), which can reveal complex relationships between dependent variables and confounding factors (Bjorklund et al. 2019; Li et al. 2017; Zheng et al. 2020). However, the GLM has some limitations, including its inefficiency in investing in the overall effect, interaction effects, potential multicollinearity problems, and non-linear exposure-response relationship. Bayesian kernel machine regression (BKMR) model has been investigated in the environmental co-exposure factors to health effects (Bobb et al. 2018; Hu et al. 2021; Li et al. 2021). It not only has a good identification effect on the major environmental factors that harm health but also can use the kernel function to estimate the relationship between the overall effect generated by joint exposure and the nonlinear exposure-response relationship and find meaningful conclusions that are difficult to find by GLM (Bellavia et al. 2019; Bobb et al. 2018; Bobb et al. 2015).

Based on the above findings, the study recruited 275 retired elderly people as the research object in Beijing, China. Then the study analyzed the effects of urinary element concentration on fasting blood glucose (FBG) level using the GLM and BKMR model and compared results from both analysis models.

\section{Materials And Methods}

\section{Research Design and Study Population}

A cross-sectional study design was implemented. The retired elderly people (aged above age 60 or more) recruited from the community-based in Beijing, in 2016. This study was approved by the Ethics Committee of the Institute of Basic Medical Sciences, Peking Union Medical College (Beijing, China), and all participants had signed informed consent before the interviews.

Personal information was collected using a structured questionnaire through face-to-face communication by the investigator, including gender, age, smoking status, alcohol drinking status, and body mass index (BMI). Medical records were used to gather information on whether the subjects had diabetes and medication use related to blood sugar control.

The vein blood $(4 \mathrm{~mL})$ was collected from each subject after overnight fasting and used to measure FBG. Blood samples were collected in vacuum tubes containing a thixotropic gel barrier (BD Vacutainer SST II, REF 367988). FBG was measured using the hexokinase method on an autoanalyzer (Roche Cobas, Germany, Baron). Morning urine samples were collected and placed in polyethylene centrifuge tubes for the measurement of the urine creatinine using an enzymatic method (Roche Cobas, Germany, Baron). After delivery to the laboratory, the urine samples were repackaged and immediately stored at $-80^{\circ} \mathrm{C}$. 


\section{Determination of Urine Elements}

A Nexion 300D (Perkin-Elmer SCIEX, USA) inductively coupled blood-mass spectrometry (ICP-MS) was used for the analysis of urine samples, operating conditions was detailed in Tables S1. The detected elements included Aluminum (Al), Chromium (Cr), Manganese (Mn), Iron (Fe), Cobalt (Co), Nickel (Ni), Copper (Cu), Zinc (Zn), Arsenic (As), Selenium (Se), Strontium (Sr), Cadmium (Cd), Cesium (Cs), Barium $(\mathrm{Ba})$, and Lead $(\mathrm{Pb})$. Urine samples were thawed at room temperature before use, then were diluted 1:10 with $0.5 \%(\mathrm{v} / \mathrm{v})$ nitric acid $+0.02 \%$ Triton $\mathrm{X}-100$ into $15 \mathrm{~mL}$ polypropylene centrifuge tubes and treated by sonication in an ultrasonic water bath for $1 \mathrm{~h}$, at $60^{\circ} \mathrm{C}$. The analysis method was referenced from previous studies, using ICP-MS with Dynamic Reaction Cell (DRC) technology (Brodzka et al. 2013; Heitland and Koster 2006; Ivanenko et al. 2013), the conditions of DRC was detailed in Tables S2. The DRC was applied to attenuate interfering background ion signals via ion-molecule reactions and improved the limit of detection (LOD) compared with the published literature (Feng et al. 2015a; Goulle et al. 2005; Wang et al. 2020b). The LOD for the element was calculated using 3 times the standard deviation of the blank signal, the LODs and DRC conditions were shown in Table S3. Rhodium (Rh) as the internal standard was simultaneously introduced via a T-piece.

\section{Quality Control}

A questionnaire information survey was conducted by strictly trained investigators with a medical background. EPI Data software was used for double parallel input to establish a database to ensure accurate and reliable input of questionnaire information. After on-site pretreatment, biological samples were immediately sent to the Clinical Laboratory Center of Peking Union Medical College Hospital. Urine samples were stored at $-80^{\circ} \mathrm{C}$ until further analysis. All polypropylene centrifuge tubes used to store urine were soaked in $10 \%$ nitric acid solution for $48 \mathrm{~h}$ and rinsed with ultra-pure water before use. For each urine element analysis, sample was measured 3 times and the mean was calculated for data analysis. The trace elements urine quality control samples and the spiked recovery samples were measured every 20 specimens to assure correct analysis of the studied specimens. The intra-assay coefficients and the interassay coefficients of variation were less than $10 \%$, Table S3. If quality control samples were not in the range (e.g.: recovery rate was not in the range of $80 \%-120 \%$ ), specimens were retested.

\section{Statistical Analysis}

Means with standard deviations were used to describe normal distribution data, and the interquartile range (IQR) and median were used to describe data with skewness distribution. A $p$-value $<0.05$ indicates statistical significance. Urinary concentrations below the LOD were assigned with LOD/2 for calculations. Element detection rates of $<60 \%$ were excluded from the statistical analysis. All skewed distribution variables were logarithmically converted into approximate normal distribution before analysis.

First, the generalized linear model was used to explore the association between each element concentration and FBG level. Element concentrations were logarithmically transformed as independent variables and FBG as the dependent variable used to establish regression equations. Covariates were 
collected based on prior knowledge and published literature. The confounding factors such as age, gender, education, smoking status, alcohol drinking status, and BMI were adjusted.

To further investigate the potential nonlinear, non-additive, and interactive relationship between urine joint exposure to co-existing elements and FBG levels established by the BKMR model, the following modeling was used.

$\mathrm{Y}_{\mathrm{i}}=\mathrm{h}\left(\mathrm{h}_{1}+\mathrm{h}_{2}+\ldots \mathrm{h}_{\mathrm{i}}\right)+\beta^{\top} \mathrm{Z}_{\mathrm{i}}+\varepsilon_{\mathrm{i}}$

In this formulation, where $h_{i}$ represents given elements in urine samples, $\beta$ represents the effect of the covariates (note that $Z_{i}$ is a vector), the BKMR model was adjusted for the same set of covariates as the generalized linear model, $\varepsilon_{\mathrm{i}} \sim \mathrm{N}\left(0, \sigma^{2}\right)$ are residual error terms. The function $\mathrm{h}()$ is a dose-response function, which contains nonlinear and/or interactions between components. The Gaussian kernel was employed as the kernel function for specifying the BKMR model because relevant studies demonstrated that the Gaussian kernel performed well across a range of plausible expose-response relationships for environmental health research (Bobb et al. 2018; Bobb et al. 2015; Valeri et al. 2017).

BKMR model analysis was performed using a two-phase modeling strategy, considering that the sample size was not very large. In the first stage of BKMR model fitting, all of the 15 elements were included in the model with the default tuning parameters for Markov Chain Monte Carlo (MCMC) processes for 10000 iterations and kept the last 5000 samples, and model convergence was visually inspected using trace plots. Posterior inclusion probabilities (PIPs) were calculated to quantify the relative importance of each element to the FBG level, whose values ranged from 0 to 1 . Subsequently, elements with PIPs $>0.1$ in the first stage analysis were entered into the next step BKMR model. The second stage BKMR analysis was combined using the same Gaussian kernel function, analysis process, and model convergence conditions. Finally, the present study showed the results from the second stage BKMR model analysis. (1) In order to illustrate the cumulative effect of the overall urinary elements estimated exposure on FBG, the BKMR model integrated the exposure levels of all elements included into one total variable and mapped the relationship between the overall element exposure estimated values and FBG at different exposure levels. (2) To aim for the association of single element exposure-response relationship on FBG and controlling the influence of other co-existing elements at their median level. (3) To quantify the potential interactive effect of co-existing elements on single element exposure-response relationship. BKMR analyzed the association between each single element changing from the 25th to 75th percentile and FBG, at varying levels of the co-existing elements have been fixed at the 25th, 50th and 75th, respectively. (4) Finally, we also visualized the bivariate exposure-response relationship for each given element (column element) and another element (parallel element) to reveal any two elements possible interactions, where the parallel element was fixed at the10th, 50th, or 90th percentile and all of the other elements were fixed at their median value.

The generalized linear models were carried out in Rstudio software (R version3.6.2) and with package "ggplot" and "bkmr" for plotting the quantify and visualize results of the BKMR model. 


\section{Results}

\section{General Characteristics of the Subjects}

A total of 275 subjects who completed interviews and biological samples collection were included in the study. From Table 1, the demographic characteristics of the subjects showed that the median age was 68.9 years old. The subjects included 122 males and 153 females, and there was a slightly greater proportion of females (55.6\%) than males. The mean BMI was $24.5 \mathrm{~kg} / \mathrm{m}^{2}$. Most of the subjects did not have long-term smoking and drinking habits.

\section{Result of Urinary Elements Analysis}

The concentrations of 15 element in the subjects' urine samples were skewed distribution, so the data was presented as a median and interquartile range in Table 2. ICP-MS analyses revealed the concentrations of most elements in the samples were higher than the detection limits. Among them, the concentrations of $\mathrm{Co}, \mathrm{Cu}$, and $\mathrm{As}$ in one sample were lower than the detection limits, The concentrations of $\mathrm{Cd}, \mathrm{Ba}, \mathrm{Pb}$, and $\mathrm{Mn}$ were below the detection limits in 15,8,39 and 105 samples, respectively.

\section{Results of the Generalized Liner Model}

Results from the generalized linear model addressing the association between 15 urinary element concentrations and FBG levels were presented in Table 3. After controlling potential confounding factors, the analysis showed that both $\mathrm{Zn}$ and Sr were a significant positive correlation with FBG levels. The FBG level was increased by 0.122 ( $\beta$ 95\% Cl: $0.122(0.073,0.171), p<0.05)$ per, 1 -unit increase in the logtransformed concentration of $\mathrm{Zn}$, and by 0.054 ( $\beta$ 95\% Cl: $0.054(0.009,0.099), p<0.05)$ per 1 -unit increase in the log-transformed concentration of Sr, respectively. At the same time, a significant inverse association was found between the concentration of $\mathrm{Cd}$ and FBG. The FBG level was decreased by 0.033 $(\beta$ 95\% Cl: $0.033(-0.060,-0.006), p<0.05)$ per 1 -unit increase in the log-transformed concentration of $\mathrm{Cd}$.

\section{Results of the Bayesian Kernel Machine Regression Model}

In the first stage of BKMR model fitting, all of the 15 elements were included. The result of trace plots showed that the BKMR model was convergent. First, BKMR calculated PIPs, which rank each element by importance to the effect in the co-existing elements, showed that part of the 15 element concentration changes had contributed little to the effect on FBG. Therefore, 8 elements (Cd, Se, Al, $\mathrm{Fe}, \mathrm{Cr}, \mathrm{Ni}, \mathrm{Mn}$ and $\mathrm{Zn}$ ) of PIPs $>0.1$ were selected and included in the second stage BKMR model. The post-MCMC analysis by inspecting the trace plots suggested that the second stage BKMR model was convergent too. $\mathrm{Cd}$, Se, $\mathrm{Al}, \mathrm{Fe}, \mathrm{Cr}, \mathrm{Ni}, \mathrm{Mn}$, and $\mathrm{Zn}$ had higher PIPs value $(1.00,0.46,0.79,0.42,0.34,0.91,0.75,1.00)$ than the first stage BKMR model, which suggested higher associations with FBG level. Then the second stage BKMR model analysis results were visualized as follows. (1) In general, the overall estimated exposure of 8 elements joint association analysis was positively correlated with the FBG level and was statistically significant when overall element concentrations were at their 65th percentile, as compared to when 
overall elements were at their median values (Fig. 1). (2) In order to analyze the nonlinear exposureresponse relationship between the single element and FBG level under control influenced by other coexisting elements, the univariate exposure-response relationship between each element and FBG level was estimated by BKMR model. Fig. 2 showed a suggestion of approximately linear effects of $\mathrm{Cd}$ and $\mathrm{Zn}$ in urine on FBG, with increasing the concentration of urinary Cd, FBG level decreased, while with increasing the concentration of urinary Zn, FBG level increased, respectively, where all of the rest of elements were fixed to 50th percentile. (3) Further BKMR analysis elaborated the relationship between single or combined elements exposure and FBG. Fig. 3 showed the variation in the association of each element with FBG level when the single element increased from 25th to 75th percentile accompanied by the other co-existing elements set at different thresholds (25th, 50th, or 75th percentile) in elderly people. It was evident from Fig. 3 that urinary $Z n$ and Cd showed a significantly association with FBG levels. A change in urinary $\mathrm{Zn}$ log-transformed level from the 25th to the 75th percentile demonstrated a significant positive in FBG of $0.197(0.141,0.253), 0.247(0.192,0.302)$, and $0.274(0.204,0.344)$ when coexisting elements were fixed at the 25 th, 50 th, and 75 th percentiles, respectively. Likewise, a change in urinary $\mathrm{Cd}$ log-transformed level from the 25th to the 75th percentile demonstrated a significant negative in FBG of $0.103(-0.147,-0.059), 0.147(-0.186,-0.108)$, and $0.187(-0.246,-0.128)$ when co-existing elements were fixed at the 25th, 50th, and 75th percentiles, respectively. (4) To further explore the possible interaction between co-existing elements, bivariate cross-sections of exposure-response curves were mapped for each element and FBG. Fig. 4 visually displayed the exposure-response curves for a given single element (column element) and FBG level when one other element (parallel element) was fixed at the 10th, 50th, or 90th percentile and all other elements were fixed at the 50th percentile. It could be seen from the results that there were some interaction relationships among some elements, such as $\mathrm{Zn}$ and $\mathrm{Cd}, \mathrm{Zn}$ and $\mathrm{Ni}, \mathrm{Mn}$ and $\mathrm{Al}, \mathrm{Cd}$ and $\mathrm{Al}$, etc.

\section{Discussion}

\section{Difference between GLM and BKMR model Analysis}

In this cross-sectional study, the association between urinary element and FBG level was analyzed by a traditional generalized linear model and a novel nonparametric BKMR model in elderly people. Both analysis models revealed similar results that Zn was significantly positively correlated with FBG, and Cd was negatively correlated with FBG, which these associations remained statistically significant after adjusting for potential confounding factors. The result was consistent with epidemiological literature, and laboratory studies provide biological evidence to support the finding. Epidemiological studies suggested that $\mathrm{Zn}$ concentration in vivo strongly correlated with glucose level. Low Zn levels in T2DM might be the result of enhancing excretion of urinary $Z n$. A research-based on the Chinese population illustrated that urinary $\mathrm{Zn}$ was associated with altered FBG, impaired fasting glucose (IFG), and diabetes risk (all $p<0.05$ ) (Feng et al. 2015b). In a separate study, serum Zn and urine Zn were measured in healthy, prediabetes, and diabetes patients from Northeast China. The serum Zn concentration was lower in T2DM and urinary $\mathrm{Zn}$ was higher in T2DM, compared with health, respectively (Xu et al. 2013). A meta-analysis based on 14 cohort studies also found that higher total Zn intake was associated with lower FBG level (Kanoni et al. 
2011). Meanwhile, laboratory research demonstrated that $\mathrm{Zn}$ was widely distributed in vivo and had important physiological functions in maintaining glucose homeostasis and glucose metabolism (Morais et al. 2019). Zn could regulate the process of glucose metabolism by participating in the synthesis of key enzymes like a-glucosidase, phosphofructokinase (PFK), pyruvate kinase (PK), and glycogen synthase (Ranasinghe et al. 2015). Moreover, Zn could modulate several signaling pathways, and part of these played crucial roles in regulating insulin production and insulin signaling pathway (Cruz et al. 2018; Fukunaka and Fujitani 2018; Norouzi et al. 2018). Zn was also reported to be an insulin-mimetic, an effect that is possibly mediated by Zinc-a2-glycoprotein. Zinc-a2-glycoprotein has emerged as a potential marker of insulin resistance in T2DM. Recent studies suggested that Zn levels were significantly correlated with oxidative stress and inflammation progression in diabetes (Hojyo and Fukada 2016). Past studies suggested that altered Zn levels activate nuclear factor-KB (NF-KB) and NLR family pyrin domain containing 3 (NLRP3), leading to the production of interleukin (IL) $-1 \beta, I L-6, I L-8$, and tumor necrosis factor (TNF) - $a$ (Bonaventura et al. 2015; Ibs and Rink 2003; Summersgill et al. 2014). These factors were implicated in the occurrence and development of diabetes (De Souza Bastos et al. 2016; Guo et al. 2015; Hang et al. 2014). Taken together, it had been hypothesized that $Z n$ deficiency in vivo was associated with the etiology of T2DM.

Both models' analysis results showed a significant negative correlation between urinary Cd and FBG level in elderly people. Cd is known to have a negative impact on human health (Tinkov et al. 2017). Although numerous epidemiological studies have focused on the association between Cd and FBG level, results have been inconsistent (Anetor et al. 2016; Edwards and Ackerman 2016; Guo et al. 2019; Wu et al. 2017). $\mathrm{Cd}$ induces adverse effects on different organs. However, kidneys remain the principal target organ (Das et al. 2019). Laboratory studies confirmed that when the concentration of $\mathrm{Cd}$ in the body was low, it mainly accumulated in the proximal tubule cells, which are mainly located in the renal cortex. The classical view was that when the concentration of Cd sustained increase approaching to about 150-200 $\mu \mathrm{g} / \mathrm{g}$, a critical threshold, the cells undergo oxidative stress that leads to injury and either necrotic or apoptotic cell death (lyama et al. 2020; Liu et al. 2009). The corresponding critical urinary Cd concentration is usually about 2-10 $\mu \mathrm{g} / \mathrm{g}$ creatinine (Jarup 2002; Roels et al. 1979). Several studies revealed that urinary levels of $\mathrm{Cd}$ in non-occupational exposed people are usually below $0.5 \mu \mathrm{g} / \mathrm{g}$ creatinine (Prozialeck and Edwards 2010). Therefore, it is necessary to conduct further studies.

The results from the second stage BKMR model analysis showed the association of independent and joint exposure between co-existing elements ( $\mathrm{Cd}, \mathrm{Se}, \mathrm{Al}, \mathrm{Fe}, \mathrm{Cr}, \mathrm{Ni}, \mathrm{Mn}$, and $\mathrm{Zn}$ ) with FBG levels in elderly people. The association analysis showed an upward trend between the overall estimated joint exposure of 8 elements with the FBG level, which was statistically significant when all element concentrations were at their 65 th percentile. For other quantiles, the associations were not significant. Probably because urine contains both beneficial and harmful elements with inhibitory or synergistic interaction with each other.

However, there were some differences in results between GLM and BKMR model. The significant positive association between urinary Sr and FBG level was only observed in GLM. There have been few epidemiological studies and biological studies about the correlation between urinary Sr and FBG level. A 
prospective study based on the Dongfeng-Tongji (DFTG) cohort, in China, found no significant association between $\mathrm{Sr}$ and diabetes incidence after adjusting for confounding factors using conditional logistic regression models. Sr concentration was not significant between the cases and control groups (Yuan et al. 2018). The association was not statistically significant in the BKMR model, probably because BKMR not only controlled confounding factors included in GLM but also evaluated the association between given elements and FBG levels in the presence of multiple elements. Therefore, the BKMR model may reveal the exposure-response relationship between element and FBG level more comprehensively.

\section{Uncertain Association between Other Urinary Elements and FBG}

Both the GLM and the second stage BKMR model analysis did not observe the evidence of the significant association between the concentrations of other elements ( $\mathrm{Se}, \mathrm{Al}, \mathrm{Fe}, \mathrm{Ni}, \mathrm{Mn}$, and $\mathrm{Cr}$ ) and $\mathrm{FBG}$ in elderly people. First, laboratory evidence suggests that maintaining appropriate levels of $\mathrm{Cr}$, Se, and $\mathrm{Mn}$ in vivo is beneficial for insulin function and glucose metabolism (Dubey et al. 2020; Li and Yang 2018; Vincent 2019). However, the results of numerous epidemical studies results were inconsistent. The reasons for those discrepancies may be due to different designs and different study groups (Liu et al. 2020; Sanjeevi et al. 2018; Yin and Phung 2015). A systematic review by Kohler showed that 8 of the 13 observational studies demonstrated a statistically significant association between Se and odds for T2DM. However, later randomized clinical trials (RCTs)study reported no association (Kohler et al. 2018). Iron may directly or indirectly influence the physiology and development of T2DM via iron metabolism indicators, including transferrin, ferritin, hepcidin, and transferrin receptor (Liu et al. 2020). A cross-sectional study based on occupational exposure suggested that none of the 3 iron concentrations (lower quantile, median, and upper quantile) were independent risks for diabetes upon adjustment for conventional confounders (Liu et al. 2016). This conclusion is consistent with our findings. The toxic effects of Al are varied and can cause multi-system toxicity (Igbokwe et al. 2019). Although systemic toxicity was often observed in experimental animal models, it was hard to observe in human studies. Recent epidemiological studies in a farming village in South India reported a correlation between Al levels and diabetes. The authors speculated that it was related to the overuse of synthetic fertilizer and aluminum cooking utensils (Velmurugan et al. 2018). There was no such exposure in our study subjects. Further studies are necessary given the limited understanding of the association between these elements and T2DM.

The metabolic cycles of various chemical elements in the body vary greatly, the cross-sectional data cannot observe any differences. In addition, although all subjects showed normal creatinine levels, the urinary element concentrations remain vulnerable to the efficiency of kidney clearance. Therefore, the data of chemical element levels in urine samples collected by cross-sectional design to reflect the internal load level of elements in the body is still partially inadequate. Finally, the sample size of our study was limited, so it is necessary to increase the sample size in the next step.

\section{Conclusion}


Both GLM and BKMR model found that Zn and Cd levels were significantly associated with FPG in elderly retirees after controlling the influence of potential confounding factors and other chemical elements. The BKMR model is more suitable for analyzing the influence of joint exposure to co-existing elements on FPG. It can be used to evaluate the possible interaction effect of co-existing elements and non-linear associations on health effects to find more meaningful results. This study provided a methodological reference and experimental evidence for relevant research.

Both GLM and BKMR model found that Zn and Cd levels were significantly associated with FBG in elderly retirees after controlling the influence of potential confounding factors and other chemical elements. The BKMR model is more suitable for analyzing the influence of joint exposure to co-existing elements on FBG. It can be used to evaluate the possible interaction effect of co-existing elements and non-linear associations on health effects to find more meaningful results. This study provided a methodological reference and experimental evidence for relevant research.

\section{Declarations}

Acknowledgments This study was supported by the China Medical Board (Grant No. 15-230). The authors gratefully acknowledge assistants from all persons involved in the project.

Authors' Contributions Liu Liu, Ang Li, Chunyu Xu, Feng Han, Zhe Liu and Qin Wang performed the data collection. Liu Liu, Chunyu Xu and Qin Wang analyzed the biological samples. Liu Liu, Ang Li and Dongqun Xu analyzed the data and wrote the paper. Liu Liu, Ang Li, Zhe Liu, Donggang Xu, Qun Xu and Dongqun Xu reviewed and edited the manuscript. Dongqun Xu was the corresponding author of this paper. Donggang Xu was also the co-corresponding author of this paper. All authors read and approved the manuscript.

Ethics approval This study was approved by the Ethics Committee of the Institute of Basic Medical Sciences, Peking Union Medical College (Beijing, China).

Informed consent All participants signed informed consent before the interviews.

\section{References}

Anetor JI, Uche CZ, Ayita EB, Adedapo SK, Adeleye JO, Anetor GO, Akinlade SK. (2016) Cadmium Level, Glycemic Control, and Indices of Renal Function in Treated Type II Diabetics: Implications for Polluted Environments. Front Public Health 4:114. https://doi.org/10.3389/fpubh.2016.00114

Bellavia A et al. (2019) Urinary concentrations of parabens mixture and pregnancy glucose levels among women from a fertility clinic. Environ Res 168:389-396. https://doi.org/10.1016/j.envres.2018.10.009

Bjørklund G, Dadar M, Pivina L, Doşa MD, Semenova Y, Aaseth J. (2019) The Role of Zinc and Copper in Insulin Resistance and Diabetes Mellitus. Curr Med Chem 27(39):6643-6657. 
Bobb JF, Claus Henn B, Valeri L, Coull BA (2018) Statistical software for analyzing the health effects of multiple concurrent exposures via Bayesian kernel machine regression Environ Health 17:67 https://doi.org/10.1186/s12940-018-0413-y

Bobb JF et al. (2015) Bayesian kernel machine regression for estimating the health effects of multipollutant mixtures. Biostatistics 16(3):493-508. https://doi.org/10.1093/biostatistics/kxu058

Bonaventura P, Benedetti G, Albarede F, Miossec P (2015) Zinc and its role in immunity and inflammation. Autoimmun Rev 14(4):277-285. https://doi.org/10.1016/j.autrev.2014.11.008

Brodzka R, Trzcinka-Ochocka M, Janasik B (2013) Multi-element analysis of urine using dynamic reaction cell inductively coupled plasma mass spectrometry (ICP-DRC-MS) - a practical application. Int J Occup Med Environ Health 26(2):302-312. https://doi.org/10.2478/s13382-013-0106-2

Cruz KJC et al. (2018) Zinc and Insulin Resistance: Biochemical and Molecular Aspects. Biol Trace Elem Res 186(2):407-412. https://doi.org/10.1007/s12011-018-1308-z

Das S, Dewanjee S, Dua TK, Joardar S, Chakraborty P, Bhowmick S, Saha A, Bhattacharjee S, De Feo V. (2019) Carnosic Acid Attenuates Cadmium Induced Nephrotoxicity by Inhibiting Oxidative Stress,

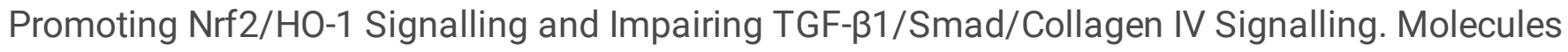
24(22):4176. https://doi.org/10.3390/molecules24224176

De Souza Bastos A et al. (2016) Diabetes and increased lipid peroxidation are associated with systemic inflammation even in well-controlled patients. J Diabetes Complications 30(8):1593-1599. https://doi.org/10.1016/j.jdiacomp.2016.07.011

Dubey P, Thakur V, Chattopadhyay M. (2020) Role of Minerals and Trace Elements in Diabetes and Insulin Resistance. Nutrients 12(6):1864. https://doi.org/10.3390/nu12061864

Edwards J, Ackerman C (2016) A Review of Diabetes Mellitus and Exposure to the Environmental Toxicant Cadmium with an Emphasis on Likely Mechanisms of Action. Curr Diabetes Rev 12(3):252-258. https://doi.org/10.2174/1573399811666150812142922

Feng W et al. (2015a) Association of urinary metal profiles with altered glucose levels and diabetes risk: a population-based study in China. PLoS One 10:e0123742 https://doi.org/0.1371/journal.pone.0123742

Feng W et al. (2015b) Association of urinary metal profiles with altered glucose levels and diabetes risk: a population-based study in China. PLoS One 10(4):e0123742.

https://doi.org/10.1371/journal.pone.0123742

Fiore M, Barone R, Copat C, Grasso A, Cristaldi A, Rizzo R, Ferrante M (2020) Metal and essential element levels in hair and association with autism severity. J Trace Elem Med Biol 57:126409. 
Fukunaka A, Fujitani Y. (2018) Homeostasis in the Pathogenesis of Diabetes and Obesity. Int J Mol Sci 19(2):476. https://doi.org/10.3390/ijms19020476

Goulle JP et al. (2005) Metal and metalloid multi-elementary ICP-MS validation in whole blood, plasma, urine and hair. Reference values. Forensic Sci Int 153(1):39-44.

https://doi.org/10.1016/j.forsciint.2005.04.020

Guo FF, Hu ZY, Li BY, Qin LQ, Fu C, Yu H, Zhang ZL (2019) Evaluation of the association between urinary cadmium levels below threshold limits and the risk of diabetes mellitus: a dose-response meta-analysis. Environ Sci Pollut Res Int 26(19):19272-19281. https://doi.org/10.1007/s11356-019-04943-3

Guo H, Callaway JB, Ting JP (2015) Inflammasomes: mechanism of action, role in disease, and therapeutics. Nat Med 21(7):677-687. https://doi.org/10.1038/nm.3893

Hang H, Yuan S, Yang Q, Yuan D, Liu Q (2014) Multiplex bead array assay of plasma cytokines in type 2 diabetes mellitus with diabetic retinopathy. Mol Vis 20:1137-1145.

Hansen AF, Simic A, Asvold BO, Romundstad PR, Midthjell K, Syversen T, Flaten TP (2017) Trace elements in early phase type 2 diabetes mellitus-A population-based study. The HUNT study in Norway. J Trace Elem Med Biol 40:46-53. https://doi.org/10.1016/j.jtemb.2016.12.008

Heitland P, Koster HD (2006) Biomonitoring of 30 trace elements in urine of children and adults by ICPMS. Clin Chim Acta 365(1-2):310-318. https://doi.org/10.1016/j.cca.2005.09.013

Henning RJ (2018) Type-2 diabetes mellitus and cardiovascular disease. Future Cardiol 14(6):491-509. https://doi.org/10.2217/fca-2018-0045

Hojyo S, Fukada T (2016) Roles of Zinc Signaling in the Immune System. J Immunol Res 2016:6762343. https://doi.org/10.1155/2016/6762343

Hu JMY et al. (2021) Prenatal Exposure to Endocrine Disrupting Chemical Mixtures and Infant Birth Weight: a Bayesian Analysis using Kernel Machine Regression. Environ Res 195:110749. https://doi.org/10.1016/j.envres.2021.110749

Ibs KH, Rink L (2003) Zinc-altered immune function. J Nutr 133(5 Suppl 1):1452S-1456S. https://doi.org/10.1093/jn/133.5.1452S

Igbokwe IO, Igwenagu E, Igbokwe NA (2019) Aluminium toxicosis: a review of toxic actions and effects. Interdiscip Toxicol 12(2):45-70. https://doi.org/10.2478/intox-2019-0007

Ivanenko NB, Ivanenko AA, Solovyev ND, Zeimal AE, Navolotskii DV, Drobyshev EJ (2013) Biomonitoring of 20 trace elements in blood and urine of occupationally exposed workers by sector field inductively 
coupled plasma mass spectrometry. Talanta 116:764-769. https://doi.org/10.1016/j.talanta.2013.07.079

lyama T et al. (2020) A novel method for assessing the renal biopsy specimens using an activatable fluorescent probe. Sci Rep 10(1):12094. https://doi.org/10.1038/s41598-020-69077-w

Jaacks LM, Siegel KR, Gujral UP, Narayan KM (2016) Type 2 diabetes: A 21 st century epidemic. Best Pract Res Clin Endocrinol Metab 30(3):331-43. https://doi.org/10.1016/j.beem.2016.05.003

Jarup L (2002) Cadmium overload and toxicity. Nephrol Dial Transplant 17 Suppl 2:35-39. https://doi.org/10.1093/ndt/17.suppl_2.35

Kanoni S et al. (2011) Total zinc intake may modify the glucose-raising effect of a zinc transporter (SLC30A8) variant: a 14-cohort meta-analysis. Diabetes 60(9):2407-2416. https://doi.org/10.2337/db110176

Kohler LN, Foote J, Kelley CP, Florea A, Shelly C, Chow HS, Hsu P, Batai K, Ellis N, Saboda K, Lance P, Jacobs ET. (2018) Selenium and Type 2 Diabetes: Systematic Review. Nutrients 10(12):1924. https://doi.org/10.3390/nu10121924

Li L, Yang X. (2018) The Essential Element Manganese, Oxidative Stress, and Metabolic Diseases: Links and Interactions. Oxid Med Cell Longev 2018:7580707. https://doi.org/10.1155/2018/7580707

Li XT et al. (2017) Association between Plasma Metal Levels and Diabetes Risk: a Case-control Study in China. Biomed Environ Sci 30(7):482-491. https://doi.org/10.3967/bes2017.064

Li Z et al. (2021) Association of multiple metals with lipid markers against different exposure profiles: A population-based cross-sectional study in China. Chemosphere 264(Pt 2):128505.

https://doi.org/10.1016/j.chemosphere.2020.128505

Liu B et al. (2016) Association of urinary metals levels with type 2 diabetes risk in coke oven workers. Environ Pollut 210:1-8. https://doi.org/10.1016/j.envpol.2015.11.046

Liu J, Li Q, Yang Y, Ma L (2020) Iron metabolism and type 2 diabetes mellitus: A meta-analysis and systematic review. J Diabetes Investig 11(4):946-955. https://doi.org/10.1111/jdi.13216

Liu J, Qu W, Kadiiska MB (2009) Role of oxidative stress in cadmium toxicity and carcinogenesis. Toxicol Appl Pharmacol 238(3):209-214. https://doi.org/10.1016/j.taap.2009.01.029

Liu M et al. (2019) Burden of diabetes, hyperglycaemia in China from to 2016: Findings from the 1990 to 2016, global burden of disease study. Diabetes Metab 45(3):286-293.

https://doi.org/10.1016/j.diabet.2018.08.008

Menke A, Guallar E, Cowie CC (2016) Metals in Urine and Diabetes in U.S. Adults. Diabetes 65(1):164-171. https://doi.org/10.2337/db15-0316 
Morais JBS et al. (2019) Association Between Cortisol, Insulin Resistance and Zinc in Obesity: a MiniReview. Biol Trace Elem Res 191(2):323-330. https://doi.org/10.1007/s12011-018-1629-y

Norouzi S, Adulcikas J, Sohal SS, Myers S (2018) Zinc stimulates glucose oxidation and glycemic control by modulating the insulin signaling pathway in human and mouse skeletal muscle cell lines. PLoS One 13(1):e0191727. https://doi.org/10.1371/journal.pone.0191727

Prozialeck WC, Edwards JR (2010) Early biomarkers of cadmium exposure and nephrotoxicity. Biometals 23(5):793-809. https://doi.org/10.1007/s10534-010-9288-2

Ranasinghe P, Pigera S, Galappatthy P, Katulanda P, Constantine GR (2015) Zinc and diabetes mellitus: understanding molecular mechanisms and clinical implications. Daru 23(1):44.

https://doi.org/10.1186/s40199-015-0127-4

Roels H et al. (1979) Critical concentration of cadmium in renal cortex and urine. Lancet 1(8109):221. https://doi.org/10.1016/s0140-6736(79)90630-5

Sanjeevi N, Freeland-Graves J, Beretvas SN, Sachdev PK (2018) Trace element status in type 2 diabetes: A meta-analysis. J Clin Diagn Res 12(5):OE01-OE08. https://doi.org/10.7860/JCDR/2018/35026.11541

Summersgill H et al. (2014) Zinc depletion regulates the processing and secretion of IL-1beta. Cell Death Dis 5(1):e1040. https://doi.org/10.1038/cddis.2013.547

Tinkov AA et al. (2017) The role of cadmium in obesity and diabetes. Sci Total Environ 601-602:741-755. https://doi.org/10.1016/j.scitotenv.2017.05.224

Valeri L et al. (2017) The Joint Effect of Prenatal Exposure to Metal Mixtures on Neurodevelopmental Outcomes at 20-40 Months of Age: Evidence from Rural Bangladesh. Environ Health Perspect 125(6):067015. https://doi.org/10.1289/EHP614

Velmurugan $\mathrm{G}$ et al. (2018) Metals in urine in relation to the prevalence of pre-diabetes, diabetes and atherosclerosis in rural India. Occup Environ Med 75(9):661-667. doi:https://doi.org/10.1136/oemed2018-104996

Vincent JB (2019) Effects of chromium supplementation on body composition, human and animal health, and insulin and glucose metabolism. Curr Opin Clin Nutr Metab Care 22(6):483-489. https://doi.org/10.1097/MC0.0000000000000604

Wang X, Karvonen-Gutierrez CA, Herman WH, Mukherjee B, Harlow SD, Park SK (2020a) Urinary metals and incident diabetes in midlife women: Study of Women's Health Across the Nation (SWAN). BMJ Open Diabetes Res Care 8(1):e001233. https://doi.org/10.1136/bmjdrc-2020-001233

Wang X, Mukherjee B, Karvonen-Gutierrez CA, Herman WH, Batterman S, Harlow SD, Park SK (2020b) Urinary metal mixtures and longitudinal changes in glucose homeostasis: The Study of Women's Health 
Across the Nation (SWAN). Environ Int 145:106109. https://doi.org/10.1016/j.envint.2020.106109

WHO (2021) Diabetes. World Health Organization (WHO)Web. https://www.who.int/healthtopics/diabetes\#tab=tab_1. Accessed 8 February 2021

Williams R (2021) IDF Diabetes Atlas 9th Edition. International Diabetes Federation (IDF)Web. https://diabetesatlas.org/en/resources/. Accessed 8 February 2021

Wu M et al. (2017) Association between cadmium exposure and diabetes mellitus risk: a prismacompliant systematic review and meta-analysis. Oncotarget 8(68):113129-113141. https://doi.org/10.18632/oncotarget.21991

Xu J, Zhou Q, Liu G, Tan Y, Cai L. C (2013) Analysis of serum and urinal copper and zinc in Chinese northeast population with the prediabetes or diabetes with and without complications. Oxid Med Cell Longev 2013:635214. https://doi.org/10.1155/2013/635214

Yin RV, Phung OJ (2015) Effect of chromium supplementation on glycated hemoglobin and fasting plasma glucose in patients with diabetes mellitus. Nutr J 14:14. https://doi.org/10.1186/1475-2891-1414

Yuan Y et al. (2018) Associations of multiple plasma metals with incident type 2 diabetes in Chinese adults: The Dongfeng-Tongji Cohort. Environ Pollut 237:917-925.

https://doi.org/10.1016/j.envpol.2018.01.046

Zheng $Y$ et al. (2020) Evaluating associations between early pregnancy trace elements mixture and 2nd trimester gestational glucose levels: A comparison of three statistical approaches. Int J Hyg Environ Health 224:113446. https://doi.org/10.1016/j.ijheh.2019.113446

\section{Tables}

Table 1 The general characteristic of subjects $(n=275)$ 


\begin{tabular}{|c|c|}
\hline Characteristic & All participants $(n=275)$ \\
\hline $\begin{array}{l}\text { Age (years), Med }\left(25^{\text {th }}-75^{\text {th }}\right) \\
\text { Gender, N (\%) }\end{array}$ & $68.9(63.0-74)$ \\
\hline Male & $122(44.4 \%)$ \\
\hline Female & $153(55.6 \%)$ \\
\hline BMI $\left(\mathrm{kg} / \mathrm{m}^{2}, \operatorname{Med}\left(25^{\text {th }}-75^{\text {th }}\right)\right)$ & $24.5(22.4-26.4)$ \\
\hline Fast Blood Glucose (mmol/L, Med(25th $\left.-75^{\text {th }}\right)$ & $5.56(4.60-5.90)$ \\
\hline \multicolumn{2}{|l|}{ Education, N (\%) } \\
\hline Illiteracy & $40(14.5 \%)$ \\
\hline Primary & $91(33.1 \%)$ \\
\hline Junior school & $55(20.0 \%)$ \\
\hline High school & $19(6.9 \%)$ \\
\hline College & $67(24.2 \%)$ \\
\hline Graduate or above & $1(0.4 \%)$ \\
\hline Missing & $2(0.7 \%)$ \\
\hline \multicolumn{2}{|l|}{ Smoking status, N (\%) } \\
\hline Non-smoking & $187(68.0 \%)$ \\
\hline Now & $42(15.3 \%)$ \\
\hline Ever & $41(14.9 \%)$ \\
\hline Missing & $5(1.8 \%)$ \\
\hline \multicolumn{2}{|l|}{ Drinking status, $\mathbf{N}(\%)$} \\
\hline Non-drinking & $157(57.1 \%)$ \\
\hline Now & $21(7.6 \%)$ \\
\hline Ever & $90(32.7 \%)$ \\
\hline Missing & $7(2.5 \%)$ \\
\hline
\end{tabular}

Table 2 Distribution of urinary element concentration among the subjects

\begin{tabular}{ccccccc}
\hline Element & \%> LOD & GM(GSD) $^{\mathrm{b}}$ & \multicolumn{4}{c}{ Selected percentiles } \\
\cline { 3 - 6 }$(\mu \mathrm{g} / \mathrm{g}$ creatinine $)$ & & & 25th & 50 th & 75th & 90th \\
\hline $\mathrm{Al}$ & 100 & $4.36(2.14)$ & 2.48 & 4.26 & 7.27 & 12.7 \\
$\mathrm{Cr}$ & 100 & $0.274(1.67)$ & 0.189 & 0.268 & 0.381 & 0.569 \\
$\mathrm{Mn}$ & 61.8 & $0.031(4.24)$ & 0.008 & 0.027 & 0.094 & 0.235 \\
$\mathrm{Fe}$ & 100 & $3.90(2.10)$ & 2.17 & 3.85 & 6.47 & 10.8 \\
$\mathrm{Co}$ & 99.99 & $0.104(2.39)$ & 0.051 & 0.105 & 0.212 & 0.338 \\
$\mathrm{Ni}$ & 100 & $0.311(3.20)$ & 0.188 & 0.375 & 0.736 & 1.08 \\
$\mathrm{Cu}$ & 99.99 & $1.08(1.75)$ & 0.749 & 1.09 & 1.60 & 2.12 \\
$\mathrm{Zn}$ & 100 & $46.8(1.78)$ & 32.6 & 47.8 & 68.3 & 103 \\
$\mathrm{As}$ & 99.99 & $2.06(2.20)$ & 1.38 & 1.95 & 3.35 & 5.23 \\
$\mathrm{Se}$ & 100 & $3.39(2.15)$ & 1.67 & 3.93 & 6.17 & 8.91 \\
$\mathrm{Sr}$ & 100 & $11.5(1.91)$ & 7.93 & 11.9 & 18.1 & 24.7 \\
$\mathrm{Cd}$ & 99.95 & $0.049(3.30)$ & 0.038 & 0.066 & 0.109 & 0.153 \\
$\mathrm{Cs}$ & 100 & $0.947(1.90)$ & 0.716 & 0.966 & 1.32 & 1.72 \\
$\mathrm{Ba}$ & 99.97 & $0.307(3.42)$ & 0.169 & 0.288 & 0.776 & 1.44 \\
$\mathrm{~Pb}$ & 99.86 & $0.118(3.83)$ & 0.042 & 0.141 & 0.325 & 0.631 \\
\hline
\end{tabular}

a \% > LOD: detection rate

${ }^{\mathrm{b}} \mathrm{GM}$ : geometric mean; GSD: geometric standard deviation.

Table 3 The association between urinary element and FBG level using the GLM 


\begin{tabular}{cccc}
\hline Element & $\beta$ & $95 \% \mathrm{CI}$ & $p$-value \\
& & & \\
\hline $\mathrm{Al}$ & 0.018 & $(-0.019,0.055)$ & 0.35 \\
$\mathrm{Cr}$ & 0.024 & $(-0.033,0.081)$ & 0.40 \\
$\mathrm{Mn}$ & 0.020 & $(-0.002,0.042)$ & 0.06 \\
$\mathrm{Fe}$ & 0.026 & $(-0.015,0.067)$ & 0.21 \\
$\mathrm{Co}$ & 0.016 & $(-0.021,0.053)$ & 0.42 \\
$\mathrm{Ni}$ & 0.009 & $(-0.022,0.040)$ & 0.57 \\
$\mathrm{Cu}$ & 0.023 & $(-0.024,0.070)$ & 0.33 \\
$\mathrm{Zn}$ & $\mathbf{0 . 1 2 2}$ & $(0.073,0.171)$ & $<0.05$ \\
$\mathrm{As}$ & 0.024 & $(-0.015,0.063)$ & 0.22 \\
$\mathrm{Se}$ & 0.027 & $(-0.018,0.072)$ & 0.24 \\
$\mathrm{Sr}$ & 0.054 & $(0.009,0.099)$ & $<0.05$ \\
$\mathrm{Cd}$ & -0.033 & $(-0.060,-0.006)$ & $<0.05$ \\
$\mathrm{Cs}$ & 0.016 & $(-0.029,0.061)$ & 0.46 \\
$\mathrm{Ba}$ & -0.003 & $(-0.028,0.022)$ & 0.82 \\
$\mathrm{~Pb}$ & -0.015 & $(-0.041,0.011)$ & 0.25 \\
\hline
\end{tabular}

Figures 


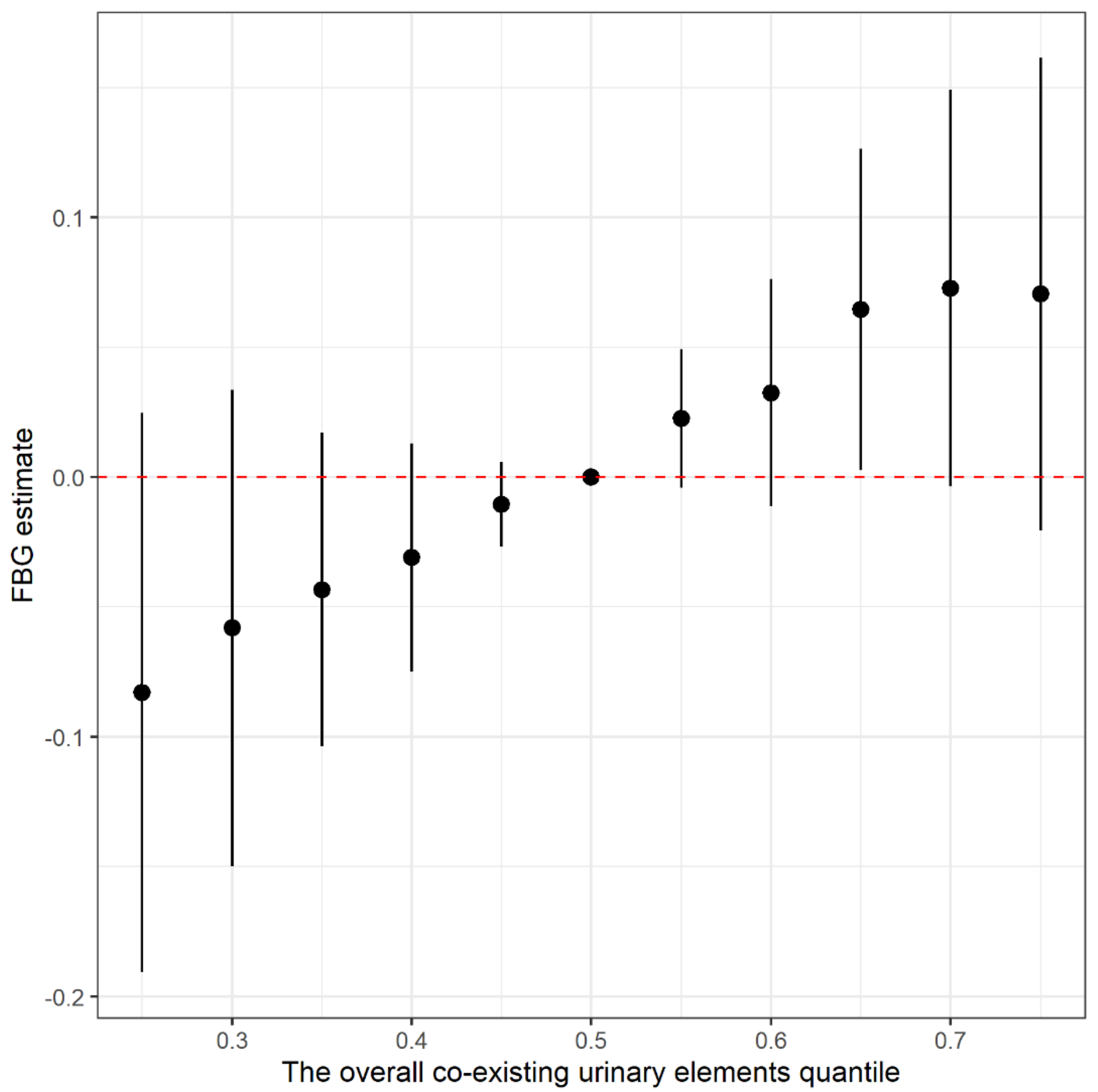

Figure 1

The exposure-response relationship between the co-existing elements overall estimated exposure and FBG in elderly people. The plots show the estimated change in overall exposure to co-existing elements (95\% credible interval) on FBG when overall exposures are all at a particular percentile (from the 25 th to 75th percentile) compared to when overall exposure estimates are all at the 50th percentile. 

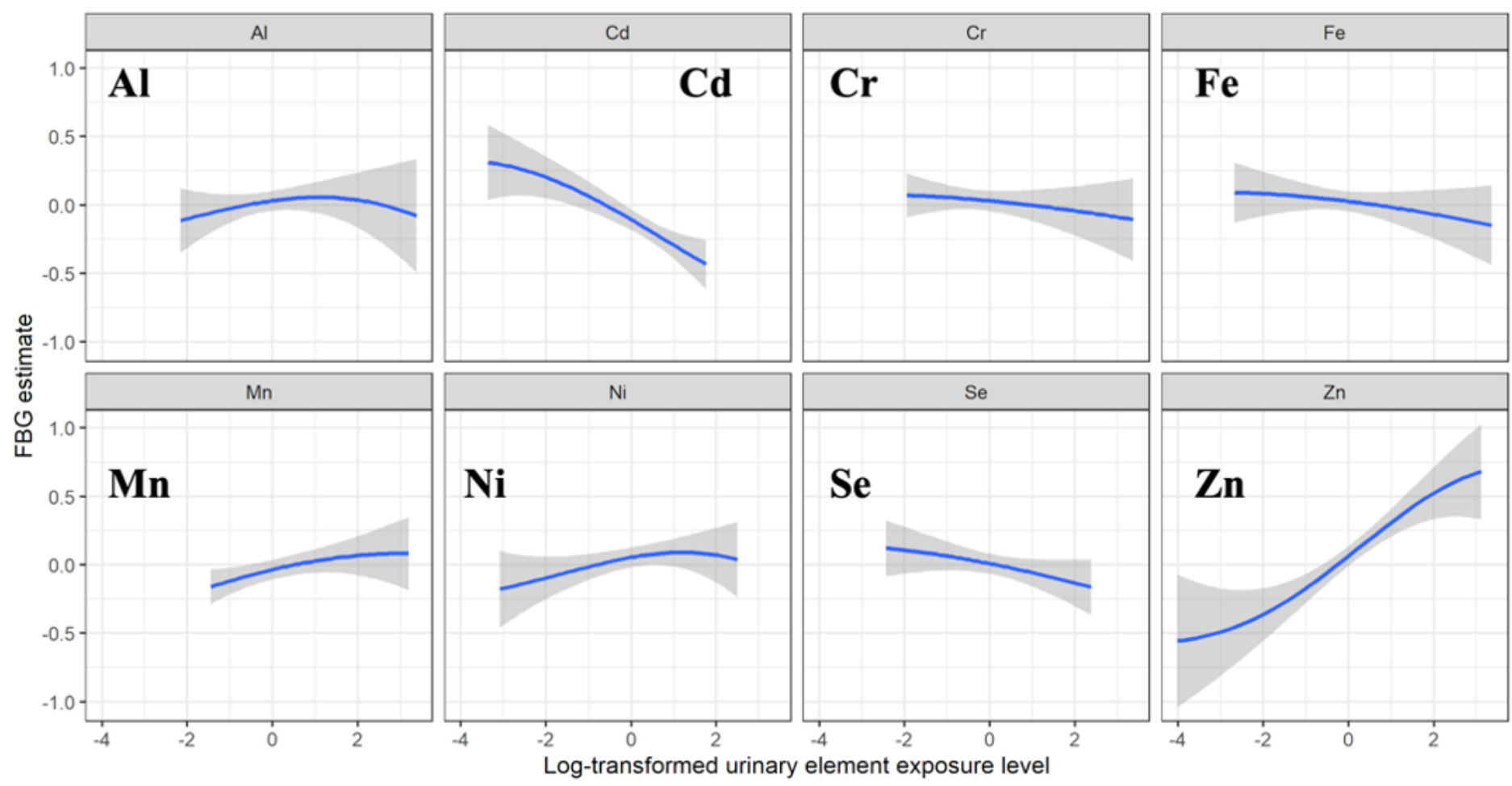

\section{Figure 2}

Univariate exposure-response curves of each element and FBG in elderly people. The plots show the association of single element exposure estimate (95\% credible intervals [Cl]) with FBG and keeping all other co-existing 7 elements at their medians.

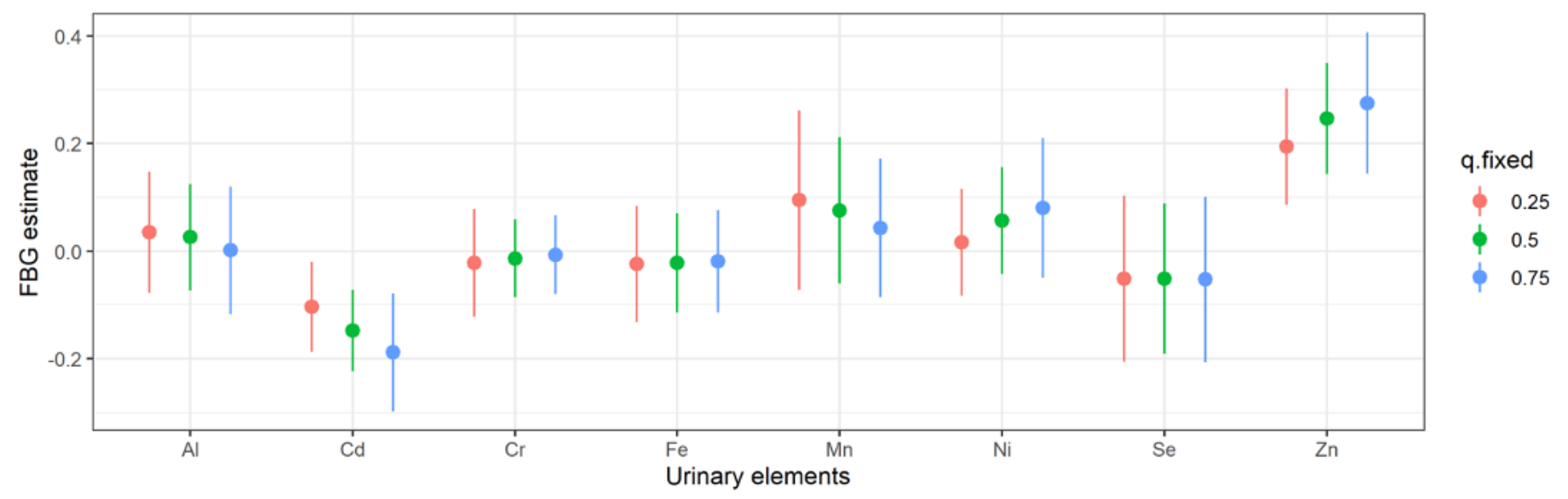

\section{Figure 3}

The association between single element exposure estimate (estimate and 95\% credible intervals) and FBG under controlling co-existing elements at different quantiles. The plots show the association between each single element changing from the 25th to 75th percentile and FBG, at varying levels of the 
co-existing elements have been fixed at the 25th (red line), 50th (green line), and 75th (blue line), respectively.

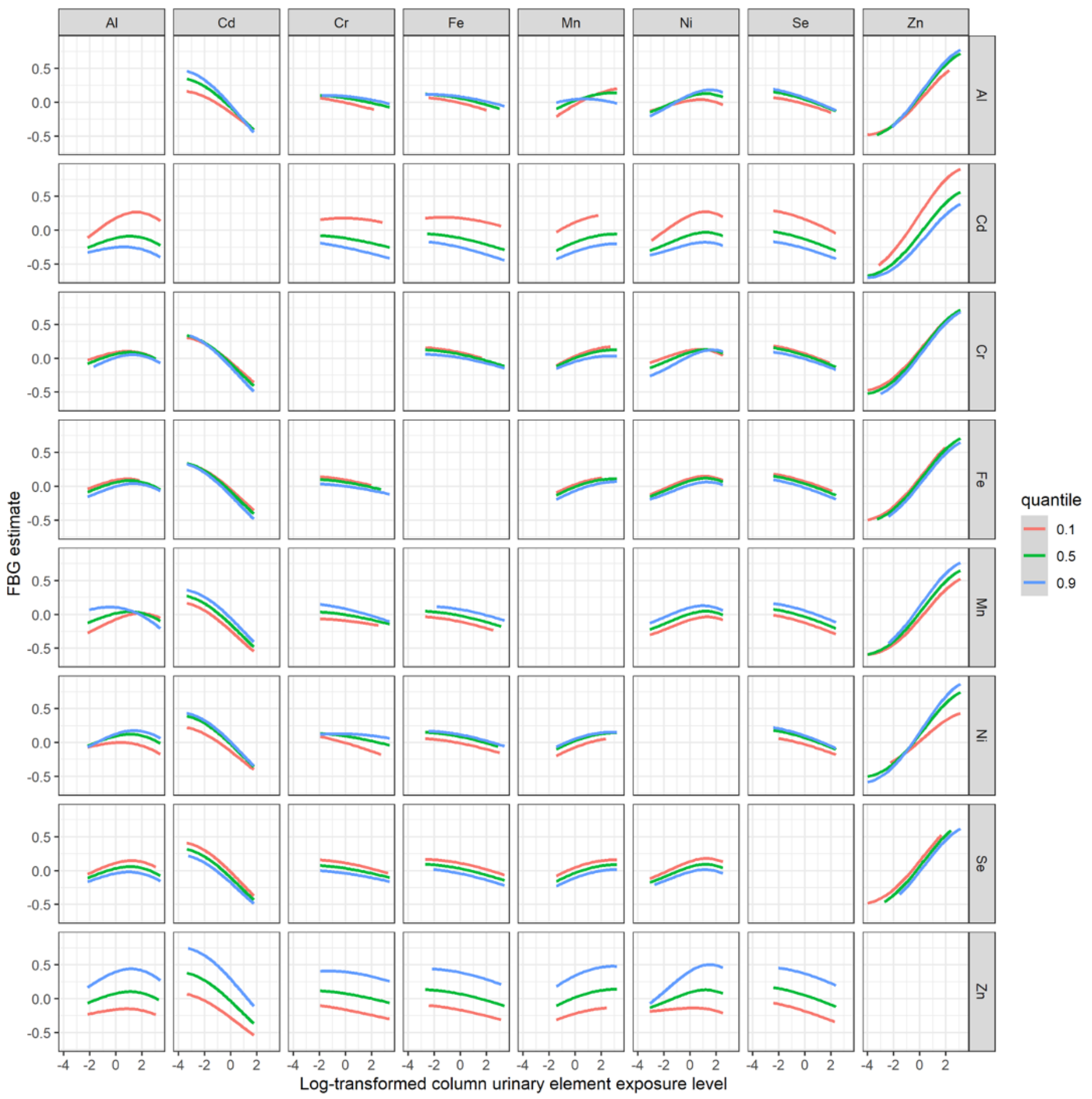

\section{Figure 4}

Bivariate exposure-response curves of each element and FBG level in elderly people. Each cell in the figure describes bivariate exposure-response curves of column element and FBG when the row element is fixed at 10th, 50th, and 90th percentiles and other 6 elements fixed at their median. 


\section{Supplementary Files}

This is a list of supplementary files associated with this preprint. Click to download.

- SupplementaryMaterial.docx 